\title{
Highly active antiretroviral therapy induced adverse drug reactions in Indian human immunodeficiency virus positive patients
}

\author{
Radhakrishnan RAJESH, Sudha VIDYASAGAR, Krishnadas NANDAKUMAR
} Received (first version): 9-Aug-2010 Accepted: 24-Dec-2010

\begin{abstract}
${ }^{*}$
Objective: To assess the incidence, severity pattern, causality, predictability and preventability of adverse drug reactions (ADRs) and to identify risk factors for adverse drug reactions in highly active antiretroviral therapy.

Methods: Enrolled patients were intensively monitored for ADRs to highly active antiretroviral therapy. Predictability was assessed based on history of previous exposure to the drug or literature incidence of ADRs. Preventability was assessed using Schumock and Thornton criteria and severity was assessed using modified Hartwig and Siegel scale. Multivariate logistic regressions were used to identify the risk factors for ADRs.

Results: Monitoring of 130 retropositive patients by active pharmacovigilance identified 74 ADRs from 57 patients. Anemia and hepatotoxicity were the most commonly observed ADRs. The organ system commonly affected by ADR was red blood cell $(21.4 \%)$. The ADRs were moderate in $77 \%$ of cases. Type A reactions (77\%) were more common. A total of $10.8 \%$ ADRs were definitely preventable. The incidence rate of ADRs (65.9\%) was highest with Zidovudine + Lamivudine + Nevirapine combination. A total of $84 \%$ interruptions to highly active antiretroviral therapy were due to toxicity. CD4 less than $200 \mathrm{cells} / \mu \mathrm{l}$, female gender and tuberculosis were observed as risk factors for ADRs.

Conclusion: Incidence of ADRs in intensively monitored patients was found to be $43.8 \%$. Anemia in HIV patients is an influential risk factor for occurrence of ADRs. With the increasing access to antiretroviral in India, clinicians must focus on early detection and prevention of ADRs to highly active antiretroviral therapy.
\end{abstract}

Keywords: Drug Toxicity. Antiretroviral Therapy, Highly Active. India.

\footnotetext{
"Radhakrishnan RAJESH. M.Pharm, Senior Grade Lecturer. Department of Pharmacy Practice, Manipal College of Pharmaceutical Sciences, Manipal University. Manipal (India).

Sudha VIDYASAGAR. MD. Professor and Head of the department of Medicine, Kasturba Medical College, Manipal University. Manipal (India). Krishnadas NANDAKUMAR. M.Pharm, PhD, Associate Professor. Department of Pharmacology, Manipal College of Pharmaceutical Sciences, Manipal University. Manipal (India).
}

\author{
REACCIONES ADVERSAS INDUCIDAS POR \\ TRATAMIENTOS ANTIRETROVIRALES \\ ALTAMENTE ACTIVOS EN PACIENTES \\ INDIOS POSITIVOS AL VIRUS DE LA \\ INMUNODEFICIENCIA HUMANA
}

\section{RESUMEN}

Objetivo: Evaluar la incidencia, gravedad, causalidad y preventabilidad de las reacciones adversas medicamentosas (RAM) e identificar los factores de riesgo de esas RAM en terapias de antiretrovirales altamente activos.

Métodos: Se monitorizó intensamente a los pacientes incluidos a la búsqueda de RAM. La predecibilidad se evaluó con base en la historia de exposiciones previas al medicamento o a la incidencia de RAM en la literatura. La preventabilidad se valoró usando los criterios de Schumock y Thornton y la gravedad se evaluó utilizando la escala modificada de Hartwig y Siegel. Se utilizaron regresiones logísticas multivariadas para identificar los factores de riesgo de RAM.

Resultados: La monitorización retrospectiva de 130 pacientes mediante farmacovigilancia activa identificó 74 RAM de 57 pacientes. Anemia y hepatotoxicidad fueron las RAM más comúnmente observadas. El sistema comúnmente afectado por las RAM fueron las células rojas sanguíneas $(21,4 \%)$.

Las RAM fueron moderadas en el $77 \%$ de los casos. Las reacciones tipo A fueron las más comunes. Un total del 10,8\% de RAM fueron definitivamente prevenibles. La incidencia de RAM más alta fue con la combinación Zidovudina + Lamivudina + Nevirapina. Un 84\% de las interrupciones de terapias antiretrovirales altamente activas fue debido a la toxicidad. Se observaron como factores de riesgo de RAM un CD4 en menos de $200 \mathrm{cel} / \mu \mathrm{l}$, el género femenino y la tuberculosis.

Conclusión: La incidencia de RAM en pacientes intensivamente monitorizados fue del $43,8 \%$. La anemia en pacientes con VIH es un factor de riesgo de influencia en la aparición de RAM. Con el creciente uso de antiretrovirales en India, los clínicos deben centrar la atención en la detección temprana y la prevención de RAM de terapias antiretrovirales altamente activos. 
Rajesh R, Vidyasagar S, Nandakumar K. Highly active antiretroviral therapy induced adverse drug reactions in Indian

human immunodeficiency virus positive patients. Pharmacy Practice (Internet) 2011 Jan-Mar;9(1):48-55 (RETRACTED BY PLAGIARISM).

Palabras clave: Toxicidad de medicamentos.

Terapia antirretroviral altamente activa. India.

This article was retracted after an investigation of plagiarism. 